\title{
Why should you commission structured patient education for adults with type 1 diabetes?
}

\author{
HELEN HOPKINSON
}

\section{Introduction}

Dose Adjustment For Normal Eating (DAFNE) is the leading structured patient education programme for adults with type 1 diabetes in the UK. Provided by a not-for-profit NHS-governed consortium, it enables patients to self-manage their disease through a skillsbased education programme. DAFNE is designed to be embedded within established NHS diabetes services. People applying DAFNE principles learn to stabilise their blood glucose, which reduces the risk of complications, improves quality of life for them and their families and reduces NHS costs.

Commissioning DAFNE will:

- Achieve cost savings of $£ 93,133$ per 100,000 population annually'

- Enable you to be compliant with the new NICE type 1 diabetes guideline (NG17)

\section{The benefits of DAFNE}

DAFNE has been shown to improve blood glucose control, to improve quality of life and to reduce the risk of severe hypoglycaemia.2,3 These improvements are associated with immediate savings in the cost of care and long-term savings through reduction in complications.

In the short term, DAFNE is associated with immediate savings from lower insulin doses and reduced hospital attendance due to ketoacidosis and hypoglycaemia. ${ }^{4}$ DAFNE training reduces progression to insulin pump therapy producing significant cost savings in reduced pump use. ${ }^{5}$ In the longer term, further savings accrue as a result of the impact of better diabetes control on diabetes complications. Diabetes is the most common cause of end stage kidney disease and preventable blindness in the UK, and the risk of these complications developing can be significantly reduced by improving blood glucose control. A Quality, Innovation, Productivity and Prevention (QIPP) case study published by NICE estimates these savings at over $£ 93,000$ per 100,000 population every year ( $£ 48$ million nationally every year). ${ }^{1}$

Greater Glasgow and Clyde Health Board, Glasgow, UK

Address for correspondence: $\mathrm{Dr}$ Helen Hopkinson Chair UK DAFNE Executive Board and Consultant Physician, Diabetes Dept, Clinic L, New Victoria Hospital, Grange Road, Glasgow, G42 9LF E-mail: helen.hopkinson@nhs.net

Br J Diabetes 2016;16:101-102

http://dx.doi.org/10.15277/bjd.2016.087

\section{Evidence}

The reliability, credibility, impact and effectiveness of DAFNE can be demonstrated through over 25 years of peer-reviewed published research in both the UK and Europe. Details of the published research can be found at www.dafne.uk.com.

\section{Patient perspective}

The improvements in blood glucose control and confidence in selfmanagement are reflected in extensive positive feedback from service users: "My bottom line in life is to function and DAFNE gives me that ability. For me, it is not a diabetic treatment, it is my life. I have control. Together DAFNE and I are delivering the best results in diabetes care I've experienced in my 25 years." - Jenny Mills Thomas, Cambridgeshire.

Over the years, peer support networks of DAFNE graduates have developed both within local services and nationally, including an active online community at www.dafneonline.co.uk.

\section{Delivering DAFNE?}

Patients are introduced to DAFNE through a five-day course delivered by your existing staff. DAFNE 'trains the trainers' and provides ongoing continuing professional development, audit of outcomes and quality assurance. There is a fixed annual contribution to support the central UK DAFNE office function, and the cost to deliver DAFNE per patient is therefore lower if more courses are delivered. Non-recurrent set-up costs include staff training, teaching aids and associated reusable resources. The cost per patient is a proportion of the annual contribution, dependent on the annual number of courses delivered. For centres delivering one course a month, this works out at approximately f50-55 per patient, including printed resources for individual patients.

Within the DAFNE portfolio there are three courses: the original one-week course delivered Monday to Friday; a five-week course delivered one day a week over five weeks; and the DAFNE pump curriculum developed for insulin pump users who never had the opportunity to complete structured education prior to getting a pump. The latter is ideal for transition patients whose parents received most of the self-management training in the paediatric service.

\section{Want to know more?}

More detailed information on the DAFNE programme and all of the research mentioned above are available at www.dafne.uk.com together with professional and user reviews of the programme. You 


\section{Case study: The Glasgow Experience Helen Hopkinson}

Specialist diabetes services are not configured to support the delivery of group programmes within their existing traditional format of 1:1 formal clinics and informal reviews. Consequently, many teams struggle to introduce high quality structured education as a core component of their type 1 diabetes service. There is a widely held belief that such education cannot be delivered without significant additional specialised staff resource. However, this is not the case and DAFNE can be delivered by existing staff through effective service redesign. Here, I describe the evolution of a cross-site structured type 1 diabetes education programme (DAFNE) within Greater Glasgow and Clyde without any increase in specialist diabetes staff resource.

In 2007 I received $£ 6,000$ from the Scottish Diabetes Group (SDG) to fund start-up of DAFNE in our centre. This paid for training for me (a consultant physician), a dietician and a diabetes specialist nurse as well as for two years of the annual DAFNE central contribution. Our DAFNE team of three reorganised our job plans to fit in the pre-course assessment, replacing some 1:1 informal patient reviews that form the backbone of traditional diabetes follow-up and which we felt might not be as effective a use of time. We found that this was effective and that the skills learnt by patients did indeed reduce the need for 1:1 attention, with reductions in severe hypoglycaemia, diabetic ketoacidosis admissions and, in most cases, a reduction in $\mathrm{HbA}_{1}$, in keeping with the published literature. DAFNE has worked most effectively for us by empowering our patients to solve their own problems and encouraging the educators to facilitate self-management rather than indulging in parental instruction.

By 2009 I was receiving requests from colleagues and directly from patients at other hospital services who were desperate to get onto a DAFNE course. We had a waiting list of 80 people from my own hospital, and it was one of the harder decisions I made not to agree to take on patients from other hospitals. Two consultant colleagues from other Greater Glasgow and Clyde hospitals decided to set up DAFNE teams and, with the managerial support of the Diabetes Managed Clinical Network, we established a DAFNE subgroup and became a single DAFNE centre. Without additional manpower resource, job redesign has enabled a sustainable programme of DAFNE course delivery and we now have DAFNE teams delivering courses at six sites within the region. We are united by the life-changing experience of hearing a person with type 1 diabetes telling you that: "You have given me my life back".

DAFNE is more than a gold standard package available to a proportion of adults with type 1 diabetes; it offers a philosophy and a set of principles which provide a core of consistency underpinning everything we do from diagnosis in type 1 diabetes. DAFNE brings clarity to the type 1 diabetes treatment pathway and patient journey because all staff are working to the same targets, using the same language and embracing the same self-management philosophy. can also contact central DAFNE for more information and for answers to specific questions at dafne@northumbria-healthcare. nhs.uk.

\section{References}

1. Department of Health. Improving the quality of care for patients with type 1 diabetes: dose adjustment for normal eating (DAFNE) 2013. Available on the NICE website at https://www.nice.org.uk/savingsandproductivity andlocalpracticeresource?ci=http $\% 3 a \% 2 f \% 2$ farms.evidence.nhs.uk\%2fresources\%2fQIPP\%2f899091\%3fniceorg\%3dtrue (last accessed 21 Feb 2016).

2. DAFNE Study Group. Training in flexible, intensive insulin management to enable dietary freedom in people with type 1 diabetes: dose adjustment for normal eating (DAFNE) randomised controlled trial. BMJ 2002;
325:746-52. http://www.ncbi.nlm.nih.gov/pmc/articles/PMC128375/ pdf/746.pdf

3. Hopkins D, Lawrence I, Mansell P, et al. Improved biomedical and psychological outcomes 1 year after structured education in flexible insulin therapy for people with type 1 diabetes: the U.K. DAFNE experience. Diabetes Care 2012;35:1638-42. http://www.ncbi.nlm.nih.gov/pmc/articles/PMC3402270/pdf/1638.pdf

4. Elliott J, Jacques RM, Kruger J, et al. Substantial reductions in the number of diabetic ketoacidosis and severe hypoglycaemia episodes requiring emergency treatment lead to reduced costs after structured education in adults with type 1 diabetes. Diabet Med 2014;31:847-53. http://onlinelibrary.wiley.com/doi/10.1111/dme.12441/epdf

5. Ray T, Choudhary P, Mansell P, et al. Dose Adjustment for Normal Eating (DAFNE) structured education reduces progression to continuous subcutaneous insulin infusion (CSII) among patients being considered for insulin pump therapy at enrolment. Diabet Med 2013;30(Suppl.1):7. 